\title{
BRONI E CASALE MONFERRATO: DUE COMUNITÀ DI FRONTE ALL'AMIANTO TRA MEMORIA, RIMOZIONE E RICONOSCIMENTO DEL DANNO
}

\author{
BRUNO ZIGLIOLI (*) \\ Nota presentata dal m.e. Marino Gatto \\ (Adunanza del 29 giugno 2017)
}

SunTO. - L'articolo compara i case study degli stabilimenti di cemento-amianto "Eternit" di Casale Monferrato e "Fibronit" di Broni. Le due cittadine sono state investite dal dramma della contaminazione da amianto, che continuerà a colpire ancora per molto tempo i lavoratori e gli abitanti. A Casale, la memoria pubblica della fabbrica e delle sue conseguenze sanitarie si è cristallizzata in modo consapevole, tanto da rendere la vicenda della cittadina emblematica sul piano nazionale e internazionale, mentre a Broni si è verificata una profonda rimozione della vicenda. Dal quadro descritto, emerge con evidenza come le lacerazioni all'interno delle comunità si aprano in un ventaglio di "visioni cittadine" che vanno dalla "ipervisibilizzazione" del problema - la fabbrica come onnipresente causa di tutti i mali della città - alla sua completa rimozione dall'orizzonte urbano, rendendo molto difficoltosi gli interventi dei pubblici poteri.

$* * *$

ABSTRACT. - The article compares the case of the Eternit cement-asbestos factory in Casale Monferrato to that of the Fibronit factory in Broni. The two towns were assailed by the drama of asbestos contamination, which will continue to affect workers and local inhabitants for a long time to come. In Casale, the memory of the factory and its consequences on the health of the inhabitants has taken the form of a concrete awareness,

(*) Professore associato di Storia contemporanea, Dipartimento di Scienze Politiche e Sociali, Università degli Studi di Pavia, Italy.

E-mail: bruno.ziglioli@unipv.it 
so much so that Casale has become an emblematic town on both national and international levels. However, the same cannot be said for Broni: here there has been a radical repression of the problem. This paper will demonstrate how tensions within the community open up a range of citizens'viewpoints, which span from a hyper-visualization of the problem - the factory as an omnipresent cause of all the evils of the city - to its complete removal from the urban horizon, making any kind of intervention on public authorities extremely difficult.

\section{LA FIBRA MIRACOLOSA}

L'amianto è un silicato a struttura fibrosa, che si trova in natura frammisto ad altri minerali (la cosiddetta "rocciamadre"), dai quali deve essere separato mediante macinazione. Ne esistono molte varietà: la più diffusa, che costituisce circa il $95 \%$ del minerale estratto nel mondo, è del tipo crisotilo, o "amianto bianco"; ve ne sono poi altre con un potenziale cancerogeno molto superiore, come la crocidolite (o "amianto blu"), l'amosite, l'antofillite e la tremolite. Si tratta di un materiale a basso costo ma dalle grandi proprietà chimico-fisiche, conosciute fin dall'antichità: risulta resistente al calore e al fuoco, all'usura, all'azione di agenti chimici e biologici, ed è fonoassorbente.

Nel corso dell'età moderna, in una fase ancora preindustriale, l'amianto venne impiegato soprattutto nel settore tessile. Una data convenzionale di inizio dell'impiego industriale dell'asbesto in epoca contemporanea può essere fissata nel 1883, quando sulla rivista inglese The Engineer comparve un articolo intitolato Asbestos and its Applications, nel quale si descrive l'uso del materiale per isolare i motori a vapore del naviglio delle marine militari britannica e tedesca: tale utilizzo si diffuse tra le flotte militari e civili di tutto il mondo a cavallo della prima guerra mondiale. Nel frattempo, nel 1901, l'austriaco Ludwig Hatschek aveva brevettato una formula per un impasto di cemento con fibre di amianto che presentava caratteristiche eccezionali di leggerezza, resistenza alle temperature e al fuoco e flessibilità di impiego: proprio per sottolineare tali qualità, il prodotto era stato ribattezzato "Eternit", mentre il brevetto svizzero divenne di proprietà dell'industriale Alois Steinmann e poi, dal 1920, della famiglia Schmidheiny, che in breve tempo realizzò un colosso industriale e multinazionale proprio incentrato sulla produzione di cemento-amianto. Le si affiancarono altri tre grandi gruppi mondiali: la Johns-Manville negli Stati Uniti, la Turner \& Newall in 
Gran Bretagna, la Cape Asbestos, britannica ma sostanzialmente radicata in Sudafrica (v. [1], pagg. 46-47 e 304-310).

Negli anni del secondo dopoguerra l'impiego del minerale e dei suoi derivati e composti si fece massiccio in moltissimi settori: nell'edilizia, nella navalmeccanica e nella cantieristica, nell'industria elettrica, chimica, aeronautica, automobilistica, ferroviaria e tessile, quale materiale isolante e di coibentazione. Alla metà degli assi settanta, l'uso di amianto in Italia e nel mondo industrializzato era massiccio, anzi: tra il 1976 e il 1980 si sarebbe toccato il culmine in termini di estrazione, produzione e consumo, in una parabola ascendente iniziata con il cosiddetto miracolo economico e industriale italiano. Non vi era quasi settore produttivo che non utilizzasse amianto nei suoi cicli di lavorazione, e nell'edilizia - residenziale, commerciale o industriale che fosse - l'amianto veniva impiegato, in particolare sotto forma di fibrocemento, nei tetti e nelle coperture, negli isolamenti termici e acustici, nei prefabbricati, nelle controsoffittature, negli intonaci, nelle canne fumarie, nelle tubazioni di abitazioni e acquedotti, nelle canalette per impianti elettrici e nelle barriere antifiamma di case e capannoni ma anche di mezzi di trasporto (navi, treni, aerei), così come nei freni delle automobili e in molti altri ambiti. Circa il 40\% degli edifici costruiti in Italia tra il 1967 e il 1975 risulta trattato con il «cemento armato dei poveri» (v. [2], pagg. 146-147).

L'impiego dell'asbesto si estese anche a oggetti di uso comune. Neppure i giochi per i bambini furono risparmiati dall'utilizzo del minerale: per esempio, una ricerca dell'Istituto per lo studio e la prevenzione oncologica dell'Azienda sanitaria e dell'Università di Firenze ha evidenziato come, tra il 1963 e il 1976, nella composizione della celebre pasta per modellare Das fosse presente amianto per il $30 \%$ circa del totale (v. [3], pagg. 80-85).

Tuttavia, parallelamente al vertiginoso aumento della produzione (v. [4], pag. 24), cominciarono a emergere le prime notizie sui pericoli connessi all'esposizione all'amianto. I risultati della prima ricerca medica rigorosa sulle malattie connesse all'asbesto, condotta dal medico inglese William E. Cooke, furono pubblicati nel luglio del 1924 dal The British Medical Journal, e individuarono una patologia a cui i lavoratori erano esposti: l'asbestosi [5]. Si tratta di una patologia cronica e progressiva da accumulo, dovuta all'inalazione delle fibre soprattutto da parte degli operai, che respiravano per molte ore al giorno, per molti anni della loro vita, la polvere di amianto. L'asbestosi comporta fenomeni di enfi- 
sema, di ispessimento della pleura e di insufficienza respiratoria e cardiorespiratoria, anche a distanza di molti anni dall'esposizione, e può avere esito mortale dopo un lungo decorso. Il primo paese a includere tale patologia nella lista delle malattie professionali fu la Germania hitleriana nel 1936, seguita dall'Italia nel 1943 e dalla Francia nel 1945. Tale riconoscimento risultò più distanziato per la Svizzera (1953), per l'Austria (1955) e per il Belgio (nel 1969).

Sempre in Germania, nel 1938, il medico Martin Nordmann descrisse alcuni casi di cancro professionale degli operai dell'amianto: a seguito di questa ricerca, il tumore polmonare fu inscritto a sua volta nel registro delle malattie professionali. Tuttavia ci vollero molti anni affinché la relazione tra l'esposizione all'amianto e l'apparizione di un mesotelioma pleurico potesse essere scientificamente provata, grazie a inequivocabili studi compiuti in Sudafrica, negli Stati Uniti e altrove tra l'inizio degli anni Sessanta e la metà degli anni Settanta (v. [6], pp. 49-58). Il mesotelioma è un cancro della pleura la cui insorgenza non è legata necessariamente a un'esposizione continuata di tipo professionale, ma anche a esposizioni più sporadiche e limitate nel tempo. Per tale ragione, non risultano a rischio solo i lavoratori che operano a contatto con l'amianto, ma anche chi vive in prossimità degli impianti, chiunque venga a contatto con fibre e manufatti, i famigliari degli operai: in altre parole, l'intera comunità. Presenta una incubazione lunghissima, superiore ai vent'anni, in media dai quaranta ai cinquant'anni: a quasi trent'anni anni dalla messa al bando della lavorazione dell'asbesto, gli epidemiologi evidenziano che nel nostro Paese il picco della patologia verrà raggiunto intorno al 2025. Saranno gli ammalati del boom dell'amianto degli anni Settanta.

L'evidenza scientifica della correlazione asbesto-mesotelioma risultava acquisita già nella prima metà degli anni Settanta. Ciononostante, la lavorazione e l'utilizzo del minerale proseguì ancora per molti anni, prima che i lavoratori, i sindacati, le comunità che ospitavano gli impianti e più in generale l'opinione pubblica prendessero coscienza del grande pericolo connesso a quel genere di produzione, e prima che le legislazioni nazionali e sovranazionali intervenissero per limitarla o vietarla. I primi paesi a dotarsi di provvedimenti in tal senso sono stati la Svezia e la Norvegia, alla fine degli anni Settanta. Nel resto dell'Europa occidentale occorrerà attendere la prima metà degli anni Novanta: in Italia il bando alla lavorazione e all'utilizzo dell'amianto fu sancito con la legge 27 marzo 1992 n. 257, dopo una difficile battaglia 
parlamentare, durata 5 anni. Molti ritardi e notevoli ambiguità si sono riscontrati sul piano comunitario: la Commissione europea ha deliberato la messa al bando dell'amianto nel 1999, e in seguito il provvedimento - entrato formalmente in vigore solo il $1^{\circ}$ gennaio 2005 - è stato parzialmente derogato nel 2009. Il Canada, per molti decenni il principale estrattore di amianto al mondo, ha interrotto l'attività delle sue miniere nel 2011. Giappone, Corea del Sud e Hong Kong hanno optato per la messa al bando completa nel 2012. In Russia (principale esportatore), India (principale importatore), Indonesia, Cina, Brasile, Messico e nella maggior parte dei paesi dell'Africa e dell'Asia l'estrazione, la lavorazione e l'utilizzo delle fibre è tutt'ora legale.

A influire su questo drammatico ritardo - drammatico in termini di vite spezzate e di gravi sofferenze - hanno contribuito una serie di fattori fra loro interconnessi, che hanno agito su diversi livelli. Senza ombra di dubbio, la scarsa comunicazione tra accademia e sapere scientifico, da un lato, e società civile, dall'altro, ha giocato un ruolo estremamente negativo. In secondo luogo, come per altri tipi di rischi sanitari e ambientali connessi alle produzioni industriali, per molto tempo le patologie asbesto-correlate sono state considerate un inevitabile prezzo da pagare in nome del progresso. In terzo luogo, dati i lunghissimi tempi di incubazione della malattia, e considerando i cicli di crescita nella produzione mondiale di amianto, la "bomba mesoteliomi" scoppiò generalmente tra la fine degli anni Settanta e la metà degli Ottanta. E solo in quel momento che il problema dell'asbesto iniziò con difficoltà a "uscire alla fabbrica" e a diventare collettivo, comune, diffuso: non più solo una questione di sicurezza sul lavoro, di tutela della salute degli operai, al limite di "monetizzazione del rischio", ma un dramma che incide nella carne viva di una intera comunità per decenni a venire. Infine, ha pesantemente influito l'attività di lobbyng messa in atto dai produttori, dal 1928 riuniti nella Saiac (Società associata industria del cemento-amianto), che per molti anni ha preferito compiere una massiccia opera di minimizzazione, puntando a promuovere un presunto «uso controllato» dell'amianto e continuando a esaltarne le caratteristiche positive, attraverso capillari campagne di comunicazione, senza informare dei gravissimi rischi correlati (v. [7], pagg. 136-138).

Certamente alla metà degli anni Ottanta nessuno poteva più considerare l'asbesto un «materiale straordinario», quanto piuttosto un lascito pesantissimo in termini umani ed economici, con le complesse procedure di sostituzione di materiali, di produzione e di bonifica a tutti i 
livelli, e soprattutto con le centinaia di Spoon River dell'amianto diffuse in tutto il mondo, in tutte le città e i paesi in cui erano insediati stabilimenti estrattivi o di lavorazione di quel minerale che fino a pochi decenni prima sembrava aver semplificato la vita di tutti.

\section{Le SPOON River DELL’AMIANTO}

Non era un problema legato solo all'asbesto e all'industria cementifera. Tra gli anni Settanta e gli anni Ottanta, l'Italia soffriva di un dissesto ambientale diffuso. I ritardi nello sviluppo industriale erano stati colmati nei decenni precedenti ricorrendo a produzioni dequalificate, a basso valore aggiunto, a basso costo della manodopera, fortemente inquinanti. La natura nella sua integralità (le acque, il suolo, l'atmosfera) e i luoghi di vita urbani erano stati completamente "mercificati" e subordinati alle priorità dell'industrializzazione: lo sfruttamento intensivo delle risorse naturali e l'insediamento di industrie fortemente impattanti a ridosso di centri densamente abitati veniva visto con favore dalla stessa opinione pubblica, tanto più nelle zone scarsamente sviluppate e con alti livelli di disoccupazione, come nel Mezzogiorno o in alcune zone depresse a economia prevalentemente rurale dello stesso Nord (v. [8], pagg. 135-140; [9], pagg. 37-41).

La grande carica cancerogena dell'amianto, la volatilità delle sue fibre, nonché la sua elevata ecletticità di utilizzo hanno reso particolarmente gravi i suoi effetti sulla salute umana, tanto da creare emergenze sanitarie di lunghissimo periodo. Intere categorie professionali sono state e sono tuttora interessate da incidenze elevate di asbestosi e mesotelioma: i militari, i marinai, gli addetti all'industria dei trasporti, della cantieristica navale e ferroviaria, gli operai siderurgici, $i$ vigili del fuoco (che indossavano tute in fibra di amianto), nonché - in particolare - tutti i lavoratori dell'edilizia e della manutenzione degli immobili, dai muratori ai lattonieri, dagli idraulici agli antennisti (che dovevano lavorare sulle ricoperture in cemento-amianto e spesso perforarle, operazione per lungo tempo compiuta senza alcuna precauzione particolare). Si tratta di un elenco del tutto parziale, proprio perché - come si è accennato - la vastità degli impieghi dell'asbesto nel campo civile e industriale ha coinvolto una miriade di attività e di prodotti anche di uso comune e ludico.

Di estrema drammaticità si è rivelata la situazione delle città che ospitavano stabilimenti destinati all'estrazione e alla lavorazione del 
minerale. Qui la contaminazione da amianto si è diffusa in tutto il tessuto urbano e ha colpito massicciamente, "nel mucchio", l'intera popolazione: non solo le maestranze che erano a diretto contatto con il minerale ma anche chi abitava a chilometri di distanza dalla fabbrica e non aveva direttamente mai avuto nulla a che fare con essa. In tali casi, il contatto con le fibre ha interferito profondamente con le strutture, le identità e le percezioni di sé delle comunità e dei centri urbani interessati. È possibile così delineare una mappa di decine di luoghi, sparsi su tutto il territorio nazionale, nei quali il contatto con l'amianto ha generato migliaia di storie di malattia, di sofferenza e di morte, che hanno finito per costituire una "narrazione urbana dell'asbesto" di forza tale da imporsi su tutte le altre narrazioni cittadine.

Casale Monferrato (Eternit), Broni (Fibronit), Grugliasco (Sia Società italiana amianto), Cavagnolo (Saca), Balangero (Società amiantifera di Balangero, che gestiva in loco la più grande miniera di amianto a cielo aperto d'Europa), Monfalcone (Fincantieri), Rubiera dell'Emilia (Eternit), Casaralta di Bologna (Officine ferroviarie), Pistoia (Breda ferroviaria), Cisterna di Latina (Goodyear), Bagnoli (Eternit), Castellammare di Stabia (Fincantieri), Bari (Fibronit), Taranto (Ilva), Priolo Gargallo (Eternit), Avellino (Isochimica); Palermo (Fincantieri): queste sono solo alcune delle città italiane segnate dal contatto con l'asbesto, e molte altre potrebbero essere citate. Da queste località, si dipanano racconti che si ripetono nella loro tragicità: gli ambienti di lavoro impolverati dalle fibre, le scarse misure di sicurezza e prevenzione, l'affacciarsi dell'infermità tra i colleghi, la persistente sensazione di pericolo spesso rimossa per il timore di perdere il lavoro, le patologie contratte dai familiari che in via indiretta venivano a contatto con il minerale, magari attraverso le tute da lavoro lavate in casa. E poi, la scoperta che la malattia usciva dalla fabbrica, colpiva gli abitanti del centro storico così come quelli delle periferie più distanti dagli stabilimenti, chi vi aveva lavorato per anni come chi vi aveva lavorato qualche settimana e chi - in termini numericamente ancora più consistenti - non vi aveva lavorato mai. Ha scritto Enrico Bullian: «[...] il mesotelioma rappresenta un'evidente epidemia, dove [...] a essere malata è la comunità ed il suo modello produttivo, non solo l'individuo» (v. [1], pag. 218).

Tale scoperta è avvenuta in modi e tempi diversi per ciascuno dei "luoghi dell'amianto", per varie ragioni che vanno dal livello consapevolezza circa le modalità del processo produttivo all'atteggiamento delle dirigenze aziendali, dal diverso ruolo giocato dai sindacati nelle 
varie realtà al contesto economico e industriale "di contorno". Tuttavia, in ogni caso, è stata accompagnata da una sorta di "congelamento" del discorso pubblico urbano, schiacciato dalla presenza dell'impianto contaminante, dalla necessità della sua chiusura e, in seguito, dal continuo monitoraggio epidemiologico e oncologico sulla popolazione, dai lutti, dalla percezione di un rischio imminente. Le stesse scelte amministrative sono risultate condizionate dall'esigenza primaria di operare la riconversione e la complessa (e costosa) bonifica del territorio: in molti casi si sono innescati duri contrasti politici e fratture tra la popolazione circa le modalità e i metodi di tali interventi.

Tra i casi più gravi di contaminazione urbana spiccano quelli di Casale Monferrato e di Broni, connotati da una altissima incidenza di mesoteliomi e di altre malattie asbesto-correlate dovuta alla presenza degli stabilimenti Eternit e Fibronit a ridosso del centro abitato. Si tratta di due casi per molti aspetti comparabili: le due cittadine distano meno di cento chilometri l'una dall'altra e si trovano in territori morfologicamente ed economicamente simili - il Monferrato e l'Oltrepò Pavese - caratterizzati dalla presenza di un territorio collinare e agricolo alle spalle, con prevalente coltura vitivinicola, e dall'apertura verso la pianura e verso alcuni centri industriali di dimensioni medio-grandi; in entrambi i casi la fabbrica, per le sue dimensioni, la sua collocazione in pieno centro urbano, la sua capacità di attrazione di manodopera, era entrata a far parte integralmente dell'identità cittadina; inoltre, i dati epidemiologici relativi ai mesoteliomi sono del tutto confrontabili. Una ricerca del 2012 ha evidenziato un'incidenza pari a 82 casi ogni 100.000 abitanti per Broni (il più elevato tasso grezzo rilevato in Italia) e di 81 casi ogni 100.000 abitanti per Casale Monferrato (v. [10], pp. 1-26).

Tuttavia, i tempi e i modi del disvelamento del rischio divergono in modo evidente, tanto fra gli attori politico-sociali preposti al suo controllo e alla sua gestione quanto fra l'insieme degli abitanti: ciò ha generato una discorde modalità di percezione del pericolo, un diverso modo di confrontarsi alla fabbrica, una differente narrazione del rapporto tra cittadini, lavoratori e amianto, un difforme processo di costruzione sociale, psicologica e memoriale dell'identità cittadina, profondamente influenzato dal contatto con la fibra e con il pericolo tuttora incombente e attuale della malattia.

Non a caso, mentre la vicenda di Casale Monferrato è diventata un simbolo a livello nazionale e internazionale degli effetti dell'esposizione all'amianto e della lotta contro le aziende responsabili, quella di Broni 
è rimasta lungamente confinata all'ambito strettamente locale e nella letteratura specialistica medica e tecnologica. Ciò si è riflesso nelle scelte politiche, nelle modalità di sviluppo e di bonifica, nonché nelle visioni future per il destino dei due centri urbani. Lo studio di tali difformità può consentire di mettere in luce alcuni aspetti che influiscono in modo determinante sulla strutturazione delle identità cittadine poste di fronte a un disastro ambientale e sanitario, attraverso l'analisi del comportamento di alcuni attori politici e sociali che svolgono ruoli chiave nella definizione di quelle medesime identità.

\section{Casale Monferrato e la Eternit}

La storia recente di Casale Monferrato è legata a doppio filo all'inizio dell'era dello sfruttamento industriale dell'amianto in Italia. Nei primissimi anni del Novecento, l'ingegner Adolfo Mazza acquistò da Ludwig Hatschek il brevetto Eternit per l'Italia e fondò nel 1906 la società Eternit Pietra Artificiale, con sede legale a Genova e stabilimento nella cittadina piemontese, sulla sponda destra del Po. La località monferrina presentava le caratteristiche ottimali per la riuscita dell'impresa: già costituiva il principale polo italiano del cemento, insieme a Bergamo, c'era acqua in abbondanza e la presenza di uno scalo ferroviario. Il terremoto di Messina e Reggio Calabria del 1908 offrì all'azienda una tragica quanto insperata occasione per decollare, con le grandi richieste di materiale per la ricostruzione che ne derivarono.

Nel 1911 l'ingegner Mazza brevettò un sistema di produzione per condutture in Eternit ad alta pressione per acquedotti che risultò molto redditizio, data la leggerezza del prodotto, la sua economicità e la sua facilità di trasporto. Gli altri manufatti dell'azienda erano costituiti da lastre e tegole per coperture, fioriere (dal 1915) e lastre ondulate (dal 1933). Nel 1917 la società venne quotata in borsa, e nel 1929 entrò a far parte del cartello Saiac. Nel 1932 lo stabilimento di Casale venne ampliato, e nel 1939 fu aperto quello di Bagnoli; dopo la Seconda guerra mondiale, furono inaugurati anche quelli di Rubiera nell'Emilia e di Priolo Gargallo, vicino a Siracusa. La produzione aumentò fino agli anni Settanta, seguendo la parabola ascendente dell'impiego dell'amianto nel mondo, e lo stabilimento raggiunse a metà degli anni Sessanta i duemila dipendenti per poi stabilizzarsi intorno ai mille fino agli anni Ottanta. 
A partire dalla metà degli anni Settanta, il consiglio di fabbrica dello stabilimento, in accordo con la locale Camera del Lavoro, iniziò una attività rivendicativa sulle condizioni di salute all'interno della fabbrica e sulle ricadute sanitarie in città. Fu una operazione non facile, che mirava a superare il concetto della "monetizzazione del rischio" per puntare alla sua limitazione e poi alla sua eliminazione: per quanto oggi possa apparire terribile, il "rischio retribuito" era considerato dagli stessi lavoratori quasi come una sorta di benefit, che consentiva di arrotondare lo stipendio con l'incentivo inserito in busta paga per coloro che operavano a diretto contatto con la materia prima. A guidare le prime rivendicazioni, talvolta anche contro l'opinione di molti lavoratori e delle organizzazioni di categoria degli edili, timorose di ricadute occupazionali, furono il giovane delegato Nicola Pondrano e l'allora segretario della Camera del Lavoro Bruno Pesce. Per esempio, venne richiesto più volte all'azienda di gestire in modo più sicuro l'attività di frantumazione degli scarti di produzione provenienti da altri stabilimenti Eternit della Penisola: i pezzi difettati venivano demoliti a cielo aperto da una ruspa, e il materiale polverizzato veniva trasportato su camion scoperti che attraversavano il centro urbano, provocando un notevole spargimento di fibre (v. [11], pagg. 47-59).

Nel 1981 il patronato Inca di Casale, diretto dallo stesso Pondrano, si impegnò in una battaglia processuale contro il diniego espresso dalla dirigenza di versare la cosiddetta "rendita di passaggio" - ovvero l'indennizzo spettante a chi aveva lavorato per anni a contatto con l'amianto - a 120 dipendenti le cui dimissioni erano state incentivate attraverso accordi e premi ad personam. Nell'ambito di tale vicenda giudiziaria, che giunse a sentenza nel 1984 con il riconoscimento delle ragioni dei lavoratori e della situazione di rischio in tutti i reparti, venne ordinata una perizia sullo stabilimento, condotta dal professor Guido Salvini dell'Università di Pavia, i cui risultati evidenziarono senza ombra di dubbio l'estrema pericolosità della produzione. Il docente ritenne di dover trasmettere gli atti della sua indagine scientifica anche alla Procura della Repubblica, affinché procedesse a ulteriori accertamenti e assumesse i provvedimenti più opportuni. Ha scritto Nicola Pondrano: «La nostra forza fu di rompere quell'accerchiamento melmoso, quell'abbraccio mortale con il quale la proprietà Eternit ci aveva avvolto per decenni: la colonia marina, la Befana per i figli, l'olio d'oliva due volte l'anno, i superminimi, lo spaccio alimentare, i turni di lavoro tarati al fine di favorire anche doppie attività (soprattutto il lavoro nei 
campi), stipendi con maggiorazioni per i lavoratori disagiati o polverosi. Tutto ciò aveva impedito, di fatto, di far nascere la consapevolezza dei pericoli che si correvano in fabbrica» (v. [12], pag. 16).

Proprio al fine di rafforzare e di rendere più efficace la battaglia giudiziaria, la Cgil di Casale ritenne di dover portare la questione dell'amianto "fuori" dalla fabbrica, coinvolgendo la comunità monferrina nel suo insieme e svolgendo opera di informazione e sensibilizzazione verso una opinione pubblica allarmata dai casi sempre più frequenti di mesotelioma (in seguito, tale patologia si rivelerà distribuita per i $2 / 3$ proprio tra $\mathrm{i}$ non dipendenti), ma che nel contempo tendeva a rimuovere e a non accogliere una realtà troppo inquietante da accettare. Così, nel 1984, l'Inca-Cgil e la Camera del Lavoro promossero a Casale un convegno sulle malattie asbesto-correlate, al quale parteciparono i più importanti studiosi del tema. Inoltre, nel 1988, su iniziativa del medesimo sindacato, venne creata una associazione delle vittime dell'Eternit (in seguito denominata Afeva, Associazione familiari vittime amianto), la cui presidenza fu affidata a Romana Blasotti Pavesi, che aveva perduto il marito, la sorella, una nipote e una figlia per mesotelioma pleurico. La nascita dello strumento associativo risultò un modo per aprirsi ulteriormente alla città, e la stessa scelta di Romana Blasotti - esterna alla Cgil - costituì un mezzo per facilitare la comunicazione con l'intero tessuto cittadino, anche nelle sue parti più critiche e diffidenti verso il sindacato di sinistra. In questo modo si produsse un "effetto di trascinamento" dell'attenzione nei confronti della comunità tutta e delle sue rappresentanze politiche di ogni colore (v. [13], pagg. 57-59).

Nel frattempo, l'Eternit aveva chiuso i battenti. Il mercato sempre più in calo, la consapevolezza che i pericoli connessi all'asbesto avrebbero a breve indotto interventi normativi limitativi, la necessità di puntare su produzioni e materiali alternativi, la mobilitazione della fabbrica e della città: sono tutte ragioni che avevano condotto a un progressivo ridimensionamento della produzione e del personale e, infine, al fallimento su autoistanza della Eternit italiana. In quel momento, a Casale vi erano ancora 350 dipendenti della multinazionale. La Safe, ovvero la holding francese del gruppo Eternit, si candidò per rilevare la fabbrica e riprendere la produzione di fibrocemento: tuttavia il sindacato oppose il proprio diniego, vincendo il prolungato tabù della difesa a oltranza dei posti di lavoro, e la stessa opinione fu espressa dai rappresentanti della cittadinanza. Il 2 dicembre 1987 - oltre quattro anni prima della legge 257/1992 - il sindaco democristiano di Casale, Riccardo Coppo, 
emanò una ordinanza che per la prima volta in Europa sanciva «il divieto, con decorrenza immediata, dell'impiego di lastre di cemento-amianto e di altri manufatti di amianto nelle costruzioni di qualsiasi genere nell'ambito del territorio comunale; il divieto dell'utilizzazione per qualsiasi uso di materiale anche residuo a precedenti processi di produzione, contenente fibre di amianto; che in caso di rimozione e smaltimento di materiali contenenti fibre di amianto le ditte esecutrici dei lavori dovranno attenersi alle prescrizioni tecnico-sanitarie che saranno disposte dalle autorità competenti» (v. [11], pagg. 78-79).

Il sindacato casalese si mostrò all'avanguardia rispetto alle confederazioni nazionali e di categoria, nella sua capacità di uscire dalla stretta logica di monetizzazione del rischio e della difesa dei posti di lavoro purchessia. Infatti in quegli anni restava diffusa la percezione del rischio ambientale quale problema esclusivamente "interno" alla fabbrica, legato alla tutela della salute dei lavoratori ma senza ricadute sul territorio circostante. Il disastro di Seveso del 1976 aveva costituito una prima occasione per un cambio di atteggiamento, contribuendo a una prima evoluzione nella condotta delle autorità politiche verso i temi ecologici. Tuttavia si trattò di un percorso lungo e accidentato: ancora per molti anni, gli stessi lavoratori dell'industria mostrarono un sentimento di ostilità verso l'ambientalismo e la salvaguardia della salute "esterna", spesso cercando di minimizzare o rimuovere rischi e danni per evitare la chiusura o il ridimensionamento delle fabbriche. Solo nella seconda metà Ottanta il movimento sindacale cominciò a porsi problemi diversi e ad allargare il campo della sua analisi a una riflessione generale sul legame tra industria, città e ambiente, andando oltre la semplice tutela dei posti di lavoro e della sicurezza interna agli stabilimenti. Si tratto comunque di un processo molto graduale: nel 1987 la stessa Cgil organizzò un convegno intitolato "Proposta ambiente", presentato come il primo incontro del genere promosso dal sindacato, dove si confrontarono esperti di ecologia di varia competenza (giuristi, economisti, medici) e rappresentanti degli interessi diffusi, come politici e sindacalisti (v. [9], pp. 39-40).

\section{BRONI E LA FIBRONIT}

Molto diversa risulta la parte finale della storia dello stabilimento Fibronit di Broni. La Cementifera italiana Fibronit SpA, con sede legale 
nella stessa Casale Monferrato, inaugurò l'impianto di Broni, a ridosso dell'abitato, nel 1919; tredici anni dopo, nel 1932, la fabbrica intraprese la produzione di cemento-amianto, con la realizzazione di condutture "a bicchiere" monolitiche, lastre ondulate, canne quadre per camini, pezzi speciali e raccorderia di completamento. Anche in questo caso nel dopoguerra la produzione aumentò, seguendo le direttrici della "fame di amianto" in Italia, e conseguentemente gli impianti vennero potenziati: negli anni Sessanta lo stabilimento lavorava su sette linee di produzione e impiegava circa 1300 operai. Negli anni Settanta, in concomitanza con il picco mondiale di produzione del materiale, furono implementate due ulteriori linee produttive. Il periodo successivo, caratterizzato dal graduale calo della domanda di asbesto, vide la Fibronit reggere apparentemente bene il colpo: la progressiva riconversione della Eternit su materiali diversi, meno inquinanti ma a maggior costo, garantiva l'acceso a un mercato "di risulta", lasciato libero dalla concorrente.

Così, la lavorazione dell'asbesto a Broni proseguì ininterrottamente fino all'approvazione della legge del 1992, e - in deroga - anche oltre. La cessazione definitiva avverrà solo nel giugno del 1993. In questo caso non si segnalano tentativi da parte del sindacato di aprire un confronto con la comunità circa i potenziali pericoli e la possibile strada di uscita dall'amianto. Nel corso degli anni Settanta anche qui il consiglio di fabbrica aveva avanzato alcune rivendicazioni per migliorare la salubrità degli ambienti di lavoro, le quali avevano portato all'installazione di filtri e aspiratori e a protocolli di lavorazione a umido meno pericolosi. Allo stesso modo, tra il 1972 e il 1993, erano state effettuate 12 indagini sull'ambiente di lavoro che avevano evidenziato molti problemi di eccessiva esposizione e di assenza di alcuni dispositivi idonei a ridurne l'impatto: l'esito di tali analisi era stato trasmesso tanto alla dirigenza quanto al consiglio di fabbrica medesimo. Tuttavia, fino alla messa al bando dell' amianto in Italia e alla successiva chiusura dello stabilimento, ciò non condusse ad alcuna azione al di fuori della fabbrica, né venne ipotizzato un possibile percorso di dismissione della produzione e di ricollocazione dei lavoratori. Eppure, la stampa e i mezzi di comunicazione avevano già ampiamente riportato le notizie provenienti dalla non lontana Casale, e lo stesso sindacato - in prima fila nella cittadina monferrina nell'individuare una via di uscita dall'amianto doveva essere in possesso di tutte le informazioni sulla pericolosità di quelle lavorazioni (v. [11], pagg 101-116). 
Racconta Osvaldo Galli, segretario della Camera del Lavoro di Voghera dal 1990 al 1998: «Chiudere le fabbriche non apparteneva alla nostra cultura. C'erano state molte chiusure tra la fine degli anni Ottanta e l'inizio degli anni Novanta, nel settore delle valigerie, nel tessile, nella meccanica, e si ritenne necessario mantenere aperta la fabbrica di Broni a ogni costo [...]. Come sindacato, non c'è stata la capacità di coinvolgere il tessuto locale, ma lo stesso può dirsi per la classe politica: non si è mai creato un sistema. Le forze politiche e istituzionali non si sono fatte carico della situazione. In Piemonte si è mossa la magistratura, da noi no, nonostante la presenza della pretura a Stradella, che non si è mai mossa, e neppure le forze politiche. Il sindacato ha tentato di tenere aperta la fabbrica, poi ha lasciato: ha sottovalutato la situazione: non siamo stati capaci di affrontare la deindustrializzazione di questa provincia». ${ }^{1}$

Romana Bianchi, deputato bronese del Pci dalla VII alla X legislatura, aveva seguito da vicino l'iter di approvazione della legge 257/1992, e racconta di un rapporto molto difficile con la città e la fabbrica: «Quando abbiamo gestito e poi approvato la legge, insieme a Bruno Pesce e al gruppo di Casale, nel sindacato a Broni c'era ancora la difficoltà a riconoscere che aver lavorato lì significava aver lavorato in un ambiente estremamente dannoso e che la chiusura della Fibronit sarebbe stata un bene per tutti. Anche tra i lavoratori della Cgil c'era, come dire, una reticenza a occuparsi di queste cose [...] Quello era, ed è ancora oggi, ancora un tabù $[\ldots]$, è come se ci fosse una rimozione $[\ldots]$. La questione vera è che a Broni non c'è mai stato, come a Casale, un movimento con una capacità di coinvolgimento di tutti i cittadini. [...]. Quando abbiamo approvato la legge, gli unici contenti erano i lavoratori vicini alla pensione, perché la legge abbuonava otto anni di anzianità. Però era come se non si dovesse parlarne troppo, della legge in via di approvazione, di amianto e di mesotelioma. È mancata una coscienza sindacale e politica capace di unificare per un obiettivo. Sembrava una fissazione di alcuni del Pci o di ambientalisti un po' snob. Agli operai gli echi di quello che accadeva a Casale non arrivavano o, se arrivavano, li rimuovevano [...]. La legge del 1992 è stata vissuta un po' come una disgrazia a Broni, e io ero il soggetto della disgrazia...». ${ }^{2}$

1 Testimonianza orale di Osvaldo Galli, Pavia, 3 giugno 2015.

2 Testimonianza orale di Romana Bianchi, Pavia, 27 marzo 2015. 
In un contesto economico connotato da un fortissimo individualismo e da una sostanziale mancanza di identità unitaria in un territorio storicamente diviso e frastagliato - con una forte resistenza al cambiamento nelle zone dell'Oltrepò più strettamente lombarde (mentre quelle più vicine al Piemonte e all'Emilia risultavano maggiormente dinamiche) - e contornato da una deindustrializzazione più rapida e di impatto maggiore rispetto ai distretti circostanti (v. [14], in particolare pagg. 107 e sgg.), il danno ambientale venne sostanzialmente rimosso. Lo stesso sindacato, in fase di regressione a livello generale, non riuscì a fare quel salto di qualità che a Casale Monferrato aveva precocemente compiuto, e verso il quale stava evolvendo a livello nazionale: restò confinato nell'azione a difesa dei posti di lavoro, e in seguito non si è neppure costituito parte civile nei processi penali contro la dirigenza della Fibronit. In questo modo, l'amianto, la fabbrica chiusa, la malattia, restarono a lungo una presenza sottaciuta ma drammaticamente penetrante, scandita dai lutti elaborati a livello del tutto individuale: «Le famiglie di Broni che sono state toccate dal problema dell'amianto, con la morte di qualche congiunto, sono la quasi totalità, alcune in maniera terribile, ma ciò non ha portato mai a una elaborazione collettiva dei lutti: si pensa che ognuno debba curarsi la propria malattia o il proprio lutto in casa propria, quasi fosse una vergogna farlo conoscere». L'amianto, rimosso a livello psicologico dal tessuto cittadino, ha finito per diventare un fattore totalizzante, in grado di condizionare la vita, lo sviluppo e l'identità della città.

\section{LO STIGMA DELL'AMIANTO}

Sulla vicenda della Eternit di Casale Monferrato sono state scritte decine di libri, con ricostruzioni di taglio giornalistico, raccolte di memorie, studi psicologici, sociologici, giuridici e sulla criminalità di impresa, analisi psicologiche sulla popolazione, eccetera (v. per esempio [15], [16], [17], [18], solo per citare alcune opere). È stato persino realizzato un fumetto, una graphic novel intitolata Eternit. Dissolvenza in bianco [19]. Nel 2013 è stato rappresentato in tutta Italia il testo teatrale Malapolvere, tratto dall'omonimo libro di Silvana Mossano [20], e a

3 Testimonianza orale di Romana Bianchi, Pavia, 27 marzo 2015. 
fine 2015 è uscito nelle sale cinematografiche il film Un posto sicuro, diretto da Francesco Ghiaccio e interpretato, fra gli altri, da Marco D’Amore, Matilde Gioli e Giorgio Colangeli [21]. Sono attivi molti progetti nelle scuole, per mantenere viva la memoria ed educare allo sviluppo sostenibile, e la cittadina monferrina è diventata addirittura meta di un "turismo responsabile" di nicchia: dopo la visita alle colline vinicole piemontesi, i turisti si recano a conoscere la storia della città che, prima in Europa, ha sfidato l'amianto.

Anche in questo caso va rimarcata la differenza con Broni. Al di là dell'attenzione del quotidiano locale La Provincia Pavese, che peraltro ha iniziato a interessarsi costantemente del problema negli ultimi dieci anni, e di alcuni articoli e reportage apparsi sulla stampa nazionale, che raccontano le storie drammatiche (queste sì, del tutto comparabili con quelle di Casale) delle vittime dell'amianto, non è stata pubblicata alcuna opera specifica, né è stato svolto alcuno studio organico, se si escludono quelli di natura medico-epidemiologica. L'interesse per la contaminazione non va oltre i confini del territorio pavese e, mentre Casale Monferrato è nota in tutta Italia per la sua tragica vicenda, quale realtà-simbolo di una produzione mortale, la vicenda di Broni è sconosciuta ai più.

A Broni il timore dello stigma dell'amianto si è percepito con particolare forza, con tutto il carico di rimozioni che spesso si accompagnano ai casi di grave contaminazione ambientale: vi ha albergato a lungo la paura, da parte della comunità cittadina, di essere classificata e riconosciuta come "città dell'amianto", anziché come "città del vino", come recita la scritta affissa a tutti gli accessi del paese (e a loro volta le preoccupazioni per le ricadute sulla produzione vincola hanno contribuito ad accrescere i processi di occultamento). La rimozione è il mezzo che si utilizza per cercare di suscitare un rapido ritorno alla normalità e per la cancellazione dello stigma medesimo: si tratta di un meccanismo non molto diverso da quello evidenziato in molte zone teatro di stragi di civili nel corso della seconda guerra mondiale. Ma alla normalità non si poteva tornare in tempi rapidi: le morti per mesotelioma continuavano a succedersi (e proseguiranno ancora per molti anni), e lo stabilimento Fibronit, un gigante strapieno di amianto su un'area di 140.000 metri quadrati, restava lì (come resta lì tuttora, seppure in corso di bonifica), come una bomba innescata che gravava sulla comunità.

Fino al 1997 non esisteva neppure una commissione amianto in seno al consiglio comunale: verrà istituita solo in quell'anno, su richiesta dei consiglieri di opposizione. Per arrivare alla costituzione di una struttura 
associativa dedicata alla lotta contro l'amianto occorre attendere il 2000, quando - su iniziativa di un gruppo di cittadini di orientamento politico molto diverso, in particolare Andrea Astranti, Domenico Novarini e Costanza Pace - venne creato un "Comitato Difesa Ambiente", che richiese alla Regione di assumere alcuni provvedimenti per la messa in sicurezza dell'area Fibronit (v. [11], pag. 128). Ma trascorsero ancora otto anni prima che si giungesse a una svolta. Gianluigi Vecchi, di Legambiente Pavia, racconta in una intervista filmata: «Quando la fabbrica è stata chiusa, l'atteggiamento della gente e degli operai, che poi erano mezzo paese, era di negare. Poi, quando hanno incominciato a esserci i primi morti, hanno rimosso. Finalmente, nel 2008 [...] noi, il sindaco, la Cgil [...], per la prima volta abbiamo parlato e abbiamo visto che la situazione era grave, e abbiamo indetto una assemblea, perché ormai le morti non era più possibile nasconderle. E lì, in quella assemblea stavolta affollatissima, finalmente si pose il problema e non fu più possibile nasconderlo». ${ }^{4}$

Solo da quell'anno iniziò a svilupparsi un tessuto associativo sul problema dell'amianto, con l'attività dell'Aiea (Associazione italiana esposti amianto), da cui poi si sono dipanati anche il Gruppo Vittime Amianto e l'Avap (Associazione vittime dell'amianto pavese), nata in quello stesso 2008 e trasformata l'anno successivo in Avani (Associazione vittime amianto nazionale). Una notevole parte dell'attività di queste associazioni è assorbita dall'organizzazione delle parti civili nei processi penali, ma hanno rivestito anche il ruolo di "imprenditori della memoria", che «selezionano, modellano e propongono definizioni del passato» (v. [22], pag. 82). In particolare, l'Avani ogni 28 aprile organizza manifestazioni in occasione della giornata mondiale delle vittime dell'amianto; promuove iniziative nelle scuole; chiede ai parenti dei deceduti di indicare sui necrologi la causa della morte; pungola gli amministratori e i politici sui temi della bonifica e dello smaltimento dell'asbesto (v. [11], pag. 129). Tale tessuto associativo, seppur di grande visibilità mediatica, è nato quando ormai la fabbrica era chiusa da quindici anni e si pone obiettivi precisi e ben demarcati, in una logica rivendicativa post novecentesca. Inoltre, riflette a livello locale le divisioni che contraddistinguono su scala nazionale i diversi sodalizi

4 Intervista a Luigi Vecchi di Legambiente Pavia, caricata su Youtube il 27 luglio 2010 nel canale del gruppo Amiantobroni: https://www.youtube.com/watch?v= GJvHMn7uU80 (visionato il 27 febbraio 2020). 
che si occupano di rappresentare le vittime dell'asbesto. Come scrive Enrico Bullian, si tratta di «un universo di piccoli gruppi non particolarmente ideologizzati [...] Le rivalità tra gruppi sono frequenti e gli obiettivi delle diverse associazioni possono divergere e scontrarsi [...]. I gruppi sono cellule autonome che costituiscono delle reti dai confini indefiniti $[\ldots] \gg$ (v. [1], pag. 321).

A Casale, già alla metà degli anni Ottanta, il sindacato si era "preso in carico" la gestione della questione amianto tra i lavoratori, l'aveva traghettata nel tessuto civile cittadino attraverso l'associazione delle vittime, l'aveva posta all'attenzione generale prospettando e negoziando le possibili vie d'uscita. Cosicché in quel caso nessuna rimozione è avvenuta, e la paura dello stigma non ha condizionato a lungo la città. Anzi, lo stigma è stato a sua volta "preso in carico" dalla comunità intera, appuntato sul gonfalone comunale, per così dire: la città ha voluto diventare un emblema della contaminazione da asbesto. La presenza precoce di una leadership in grado di assumere le responsabilità e la guida nella pianificazione e nell'attuazione dei percorsi legali ha consentito di realizzare una sorta di "resilienza", intesa come quella capacità di riconoscere il prima e il dopo del vissuto traumatico, che permette di trasformare il danno sofferto in potenzialità (v. [16], pagg. 56 e 65). Insomma, l'azione del sindacato e dell'associazione ha consentito di costruire un «quadro» della memoria collettiva, che ha permesso «la conservazione, lo sviluppo e l'esplicitazione dei contenuti della memoria dei singoli» (v. [23], pag. 22). Tali «quadri» costituiscono «il prodotto della sopravvivenza di gruppi che, nella vita quotidiana, rappresentano i punti di riferimento più immediati e familiari per il soggetto [...]. Essi propongono dei modelli di comportamento la cui struttura ed il cui significato trovano giustificazione nell'ambito di specifiche memorie collettive. Questi referenti hanno senso finché sopravvivono le identità collettive che, sull'idea di una memoria condivisa, hanno costruito i propri confini identitari» (v. [24], pag. 66).

I procedimenti giudiziari, quelli conclusi e quelli in corso, hanno costituito ulteriori momenti di identificazione della comunità nel dramma collettivo: la lotta comune contro l'amianto è diventata un elemento fondativo e unificante dell'identità cittadina, anche nei momenti più difficili e di fronte alle scelte più controverse. Invece, nel centro oltrepadano l'amianto risulta ancora oggi un elemento divisivo, che genera polemiche e contrasti nella lotta politica e tra i cittadini. Paradigmatica da questo punto di vista è la storia della bonifica dei due territori - il 
Monferrato e l'Oltrepò - nei quali l'asbesto è stato utilizzato in modo ancor più massiccio che altrove, proprio per la presenza dei due poli produttivi: tra l'altro, gli abitanti potevano approvvigionarsi direttamente presso gli stabilimenti, dove venivano venduti a prezzi molto bassi o addirittura regalati manufatti non commercializzabili perché lievemente difettati; inoltre, potevano prelevare senza formalità i sacchi contenenti il cosiddetto "polverino", ovvero la polvere di tornitura delle condutture in cemento-amianto, con la quale venivano realizzate miscele "casalinghe" per la pavimentazione di strade private, per la copertura dei fondi dei cortili, per i campi da bocce, per i sottotetti. A Casale Monferrato i piani di bonifica sono stati molto precoci e hanno portato a risultati soddisfacenti già da anni: la stessa area dello stabilimento Eternit è stata completamente bonificata nel 2005, mentre nel 2001 era stata risanata la parte di sponda destra del Po, ove un canale di scolo della fabbrica aveva creato una vera e propria spiaggia contaminata con amianto misto a sabbia. Nell'Oltrepò Pavese la bonifica è in grave ritardo, e lo stabilimento Fibronit costituisce ancora una ferita urbanistica nel tessuto urbano di Broni, fonte di continua preoccupazione per gli abitanti. Tale ritardo è dovuto anche alle difficoltà riscontrate in provincia di Pavia nell'individuare un sito di smaltimento del minerale: i comitati bronesi hanno rifiutato decisamente l'ipotesi che esso possa essere localizzato nella loro città, perché essa «ha già dato a dismisura» in termini di presenza di amianto; anche le altre soluzioni ipotizzate si sono rivelate di non agevole realizzazione, per l'opposizione degli abitanti e di molti amministratori locali. Invece, a Casale Monferrato il sito di smaltimento è stato realizzato nel 2001 nel territorio comunale, con l'accordo dell'Afeva e il confronto con i cittadini (v. [11], pagg. 130-133). Si tratta di una ulteriore dimostrazione della volontà e della capacità di "farsi carico" del problema amianto fino in fondo, senza alcuna rimozione: «La presenza della discarica di amianto a Casale è fondamentale, sotto tutti i punti di vista, anche sotto quello del riconoscimento del danno subito. Noi lo abbiamo prodotto, noi lo abbiamo usato, noi ci siamo ammalati, noi lo eliminiamo». ${ }^{5}$ E il cerchio, così, si chiude [25].

Insomma, mentre a Broni neppure i procedimenti penali sono riusciti a concorrere in maniera decisiva alla cristallizzazione di una 
coscienza e di una memoria comune degli eventi, «a Casale il sostegno politico e sociale e la partecipazione ad azioni collettive [...] hanno contribuito nelle persone alla creazione di un luogo mentale in cui confrontarsi con la propria capacità di non sentirsi completamente in balia degli eventi, anzi di poter sperimentare un'area di libertà di azione» (v. [16], pag. 69).

\section{CONCLUSIONI}

Casale Monferrato è "uscita dall'amianto" proprio perché ha trovato la forza di riconoscersi nel suo dramma, senza occultamenti o rimozioni, integrandolo nel tessuto e nell'identità comunitaria. Broni ne è ancora "dentro", proprio perché per lungo tempo non è riuscita a riconoscerlo come parte integrante, per quanto subìta e non voluta, del vivere e del morire collettivo. La rinascita di Casale, pur con tutti i problemi economici comuni a tutti i centri urbani del paese, è stata evidente mentre, per gli abitanti dei dintorni, Broni resta un luogo pericoloso, e il suo caso andrebbe ulteriormente approfondito attraverso capillari studi sociologici sul territorio. Una differenza fondamentale nelle due vicende è ravvisabile nel diverso atteggiamento assunto tra gli anni Ottanta e i primi anni Novanta dalle forze di massa intermedie, in particolare in quello del sindacato: precocemente capace di farsi carico del trade-off lavoro-salute per i lavoratori e per i cittadini nel caso monferrino, lungamente fermo sulla difesa a oltranza dei posti di lavoro nel caso bronese.

La crisi delle identità collettive ha prodotto la progressiva perdita di mordente delle organizzazioni politiche e sindacali di massa, e la loro sostituzione nel processo rivendicativo con strutture associative sul modello dei comitati, per loro natura parziali e frammentate. Tale processo ha creato un problema ulteriore, anche in relazione alla possibilità della popolazione di essere coinvolta nei processi decisionali circa il destino delle città, del loro sviluppo industriale, della pianificazione urbanistica (v. [26], pagg. 82 e sgg; [27], pagg. 63 e sgg). Da questo punto di vista, oggi il caso italiano più eclatante è certamente quello dell'Ilva di Taranto, caratterizzato da una spaccatura verticale della città, tra chi "ipervisibilizza" la fabbrica, considerandola la fonte di tutti i mali del territorio (e finendo così per trascurare quelli dovuti ad altri fattori, anche di più lungo periodo) e chi invece la "invisibilizza", 
rimuovendone gli aspetti più problematici, in nome della necessità di salvaguardare l'occupazione. Anche nella vicenda pugliese, il mondo sindacale e politico ha scontato un drammatico ritardo nella gestione complessiva dell'emergenza (v. [28], pagg. 286-290).

Ciò che emerge con chiarezza è che l'informazione e il coinvolgimento dei cittadini nelle scelte politiche e amministrative circa il destino delle città assume un'importanza ancora più forte, nel momento della crisi delle grandi ideologie e nella "società liquida" di oggi (v. [29], pagg. 427-429). Solo in questo modo le comunità possono riuscire a trasformare gli ostacoli in potenzialità. È una grande responsabilità collettiva posta in capo ai decisori, ai cittadini, ma anche ai possessori dei saperi in grado di orientare razionalmente le scelte: ovvero, a quel mondo scientifico e accademico che, conscio dei pericoli connessi all'utilizzo dell'amianto fin dagli anni Sessanta, non è riuscito a comunicarli e trasmetterli al di fuori dei suoi circoli.

\section{BIBLIOGRAFIA}

1. Enrico Bullian, Il male che non scompare. Storia e conseguenze dell'uso dell'amianto nell'Italia contemporanea, Il Ramo d'Oro, Trieste, 2008.

2. Mirco Volpedo, Davide Leporati (a cura di), Morire d'amianto. L'Eternit di Casale Monferrato: dall' emergenza alla bonifica, La Clessidra Editrice, Genova, 1997.

3. Stefano Silvestri, Francesco Di Benedetto, Corrado Raffaelli, Angela Veraldi, Asbestos in toys: an exemplary case, Scandinavian Journal of Work, Environment and Healt, anno: 2016 numero: 1 volume: 42.

4. Robert L. Virta, Worldwide Asbestos Supply and Consumption Trends from 1900 through 2003, U.S. Geological Survey, Reston-Virginia, 2006.

5. William Edmund Cooke, Fibrosis of the Lungs Due to the Inhalation of Asbestos Dust, The British Medical Journal, July 25, 1924.

6. Francesco Carnevale, Evoluzione e destino delle conoscenze sugli effetti dell'amianto per salute dei lavoratori, in: Ariella Verrocchio (a cura di), Storia/Storia di amianto, Ediesse, Roma, 2012.

7. Diego Alhaique, Fulvio Cavarian, Laurent Vogel, Amianto, tragedia senza confini, postfazione, in: Guido Iocca, Casale Monferrato: la polvere che uccide. Voci dalla Chernobyl italiana, Ediesse, Roma, 2011.

8. Saverio Luzzi, Il virus del benessere. Ambiente, salute, sviluppo nell'Italia repubblicana, Laterza, Roma-Bari, 2009.

9. Bruno Ziglioli, La mina vagante. Il disastro di Seveso e la solidarietà nazionale, FrancoAngeli, Milano, 2010.

10. Stato dell'arte e prospettive in materia di contrasto delle patologie asbesto-correlate, Quaderni del Ministero della Salute, anno: 2012 numero: 15 (maggio-giugno). 
11. Bruno Ziglioli, "Sembrava nevicasse". La Eternit di Casale Monferrato e la Fibronit di Broni: due comunità di fronte all'amianto, FrancoAngeli, Milano, 2016.

12. Nicola Pondrano, Prefazione, in: Guido Iocca, Casale Monferrato: la polvere che uccide. Voci dalla Chernobyl italiana, Ediesse, Roma, 2011.

13. Bruno Ziglioli Romana e le altre. La narrazione femminile dell'amianto a Casale Monferrato, La camera blu, anno: 2018 numero: 18.

14. Antonella Zucchella, Stefano Denicolai, Analisi strategico-organizzativa per lo sviluppo locale. Il Development Migration Path, FrancoAngeli, Milano, 2006.

15. Rosalba Altopiedi, Un caso di criminalità di impresa. L'Eternit di Casale Monferrato, L'Harmattan, Torino, 2011.

16. Antonella Granieri, Amianto, risorsa e dramma di Casale: risvolti psicologici nelle persone affette da mesotelioma e nei loro familiari, Fratelli Frilli Editori, Genova, 2008.

17. Alberto Prunetti, Amianto. Una storia operaia, Alegre, Roma, 2014.

18. Alessandro Calvi, Paracarri. Cronache da un'Italia che nessuno racconta, Rubbettino, Soveria Mannelli, 2015.

19. Gea Ferraris, Assunta Prato, Eternit. Dissolvenza in bianco, Ediesse, Roma, 2011.

20. Silvana Mossano, Malapolvere. Una città si ribella ai «signori» dell'amianto, Sonda, Casale Monferrato, 2010.

21. Un posto sicuro, regia di Francesco Ghiaccio, Italia, 2015, 102 minuti.

22. Teresa Grande, Le origini sociali della memoria, in: Anna Lisa Tota (a cura di), La memoria contesa. Studi sulla comunicazione sociale del passato, FrancoAngeli, Milano, 2001.

23. Paolo Jedlowski, Introduzione alla prima edizione, in: Maurice Halbwachs, La memoria collettiva, Unicopli, Milano, 2001.

24. Marita Rampazi, Storie di normale incertezza. Le sfide dell'identità nella società del rischio, Led, Milano, 2009.

25. Barry Commoner, Il cerchio da chiudere. La natura, l'uomo, la tecnologia, Garzanti, Milano, 1972.

26. Gian Luigi Bulsei, Ambiente e politiche pubbliche. Dai concetti ai percorsi di ricerca, Carocci, Roma, 2005.

27. Gian Luigi Bulsei, Le politiche ambientali. Intervento pubblico e regolazione sociale, Rosenberg \& Sellier, Torino, 1990.

28. Salvatore Romeo, L'acciaio in fumo. L'Ilva di Taranto dal 1945 a oggi, Donzelli, Roma, 2019.

29. Marta Dente, Giovanna Landi, Informazione e partecipazione. Il ruolo dell'opinione pubblica, in: Achille Cutrera, Giuseppe Pastorelli, Barbara Pozzo (a cura di), Seveso trent'anni dopo: la gestione del rischio industriale, Giuffrè, Milano, 2006. 